\title{
EVOLVING TAKAGI-SUGENO-KANG FUZZY SYSTEMS USING MULTI POPULATION GRAMMAR-GUIDED GENETIC PROGRAMMING
}

\author{
Athanasios Tsakonas, Bogdan Gabrys \\ Smart Technology Research Center, Dept. of Design, Engineering and Computing, Bournemouth University, Poole, UK \\ atsakonas@bournemouth.ac.uk,bgabrys@bournemouth.ac.uk
}

Keywords: Genetic Programming, Fuzzy Rule Based Systems, Evolutionary Computation

\begin{abstract}
This work proposes a novel approach for the automatic generation and tuning of complete Takagi-SugenoKang fuzzy rule based systems. The examined system aims to explore the effects of a reduced search space for a genetic programming framework by means of grammar guidance that describes candidate structures of fuzzy rule based systems. The presented approach applies context-free grammars to generate individuals and evolve solutions through the search process of the algorithm. A multi-population approach is adopted for the genetic programming system, in order to increase the depth of the search process. Two candidate grammars are examined in one regression problem and one system identification task. Preliminary results are included and discussion proposes further research directions.
\end{abstract}

\section{INTRODUCTION}

The application of fuzzy rule-based systems (FRBS) has been proven effective in a wide area of domains and problem tasks (Jang 97). Among their implementations, Mamdani-FRBS have been used for classification tasks, while regression and system identification application areas have been dominated by the Takagi-Sugeno-Kang (TSK) -FRBS. In order to exploit the power of an FRBS, commonly a training phase takes place with the neuro-fuzzy techniques most often used. In their simplest approach, these systems require the pre-determination of the rule-base size. Advanced approaches may adopt an ad-hoc or heuristic incremental process to generate the fuzzy rule base. Others make use of evolutionary techniques aiming to provide efficient rule-base generation.

Genetic programming (GP) is a successful branch of evolutionary computation effectively applied in a wide range of tasks such as symbolic regression and network design (Koza 92). In the past, GP has been successfully tested for the production of MamdaniFRBS (Alba et al. 96). Consequently, the perspective for generating TSK-FRBS within GP framework has always been regarded as a promising research field, since such a system could combine the attrac- tive properties of both methods.

In response to the need for such a hybrid, GP was effectively combined with TSK-FRBS in the past. These approaches commonly involved partial contribution of GP techniques, such as the assistance by GP in locating proper membership functions for a TSKFRBS (Hoffman and Nelles 01) or the co-evolution of several segments of a TSK-FRBS (Delgado et al. 04). In this paper, we propose an integrated approach, for the automatic design and tuning of complete TSKFRBS for regression and system identification. To accomplish this, we use grammars to describe the complete structure of TSK-FRBS within a GP individual, thus mapping one GP-individual to exactly one TSKFRBS. Using this approach we are able to generate arbitrarily sized TSK-FRBS which can be tuned using easy to formulate and implement integrated approach. We furthermore enhance our search procedure by incorporating multi-population architectures to the GP solution pool. We test two variants of this system in one prediction and one system identification task.

The content of this paper is organised as follows. Next section describes the scientific background of the methods to be used. In Section 3, the design of the system and its implementation are presented. The results and discussion related to the system effective- 
ness are included in Section 4. The conclusions and suggestions for further research are drawn in the Section 5 .

\section{BACKGROUND}

Fuzzy sets are an extension to the classic (crisp) sets, where the transition for a value from belonging to a set and not belonging to the set is gradual and quantified by a suitably chosen membership function. When performing fuzzy reasoning, we apply, in a generalized form, the traditional two-valued logic, the modus ponens. More specifically, a fact can be more or less true, based on the truth of another fact. The general expression of a rule in a Takagi-Sugeno-Kang (TSK) fuzzy rule based system has the following form (Jang 98):

$$
\begin{gathered}
R_{i} \text { : if } x_{1} \text { is } d_{k} \text { and ...then } y=f\left(x_{1}, \ldots, x_{n}\right) \text { with } C \\
\quad(i=1,2, \ldots, m),\left(x_{1}, \ldots, x_{n} \in \mathbf{X}\right), C \in[0,1]
\end{gathered}
$$

In this formula, $C$ is the certainty factor and $\mathbf{X}$ is the input set.

Genetic programming - GP (Koza 92) is a search methodology belonging to the evolutionary computation family, which in its canonical form permits the automatic generation of programs. Among successful evolutionary computation approaches, GP keeps a significant position due to its beneficial qualities, such as the production of arbitrary-sized solutions and the upholding of population diversity throughout runs.

Multi population evolutionary models, or island models, divide the population into sub-populations, often called demes, and periodically exchange a number of individuals, simulating migration. In this paper, we adopt a five island model, and we employ a canonical setup, according to literature (Fernández et al. 03).

In canonical GP, the type of nodes is implicitly defined by the number of each node's arguments (Koza 92). Hence, usually there are two types of nodes in the standard GP: a) terminals - non-function nodes; and b) functions - non-terminal nodes. In order to produce in an efficient way more complex structures within GP, such as fuzzy rule based systems, we must define a stricter hierarchy of the potential architectures. To accomplish this, one of the most efficient ways is the application of a context-free grammar (Montana 95). The standard notation to express context-free grammars is in the Backus Naur Form (BNF).

The idea to incorporate fuzzy inference into GP is not new. In (Alba et al. 96) a model that uses a context-free grammar for the production of Mamdani fuzzy rule bases has been examined. The system has proven competitive in various classification tasks. In (Delgado et al. 04), a genetic algorithm is proposed for a co-evolutionary system that produces TSK fuzzy systems. In (Chen 07), the generation of a specific class of TSK fuzzy systems is accomplished using evolutionary programming. In (Hoffman and Nelles 01 ), a genetic programming system has been used to improve a greedy algorithm (LOLIMOT) in search of data clusters by partitioning the search space.

Our approach aims at the production of TSK fuzzy rule based systems in the context of multi population grammar guided genetic programming. It has several advantages in comparison to the previous attempts. Firstly, it makes use of the genetic programming framework which has a number of desirable properties in terms of maintaining population diversity and providing arbitrary solution size. Secondly, our approach is comparatively simple to implement, since only one population is maintained, and all fuzzy rule base parameters are tuned within one system run. Finally, it is an integrated approach, that generalizes the applicability of GP for the production of fuzzy systems by generating complete TSK rule bases for regression and system identification. The structure of the TSK fuzzy system is fully described within the GP functions, and every tuning parameter is computed by evolutionary means.

\section{DESIGN AND IMPLEMENTATION}

We have evaluated the proposed system within two data domains. The first domain is the Concrete Slump Test (Yeh 08). We have retained $20 \%$ of the data as test set with the remaining $80 \%$ used for learning. The second problem addressed in this work is a system identification task. We considered the Mackey-Glass non-linear chaotic time series, as this is commonly used as a test for TSK models (Chen 07). We generated 400 data points out of which 80 were were used as the test set with the remaining data used for training.

We examined two system configurations. The first system evolves a rule base with the rules in the following form:

$$
\begin{gathered}
R_{i}: \text { if } x_{1} \text { is } \mu_{k_{1}}\left[\text { and } x_{2} \text { is } \mu_{k_{2}} \ldots\right] \\
\text { then } y=f\left(x_{c}\right) \text { with } C \\
f\left(x_{c}\right)=w_{0} x_{c}^{n}+w_{1} x_{c}^{n-1}+\ldots+w_{n-1} x_{c}+w_{n} \\
(i=1, \ldots, m),\left(x_{1}, \ldots, x_{n} \in \mathbf{X}\right), C \in[0,1]
\end{gathered}
$$

The second system evolves a rule base with the rules in the following form:

$$
R_{i} \text { : if } x_{1} \text { is } \mu_{k_{1}}\left[\text { and } x_{2} \text { is } \mu_{k_{2}} \ldots\right]
$$




$$
\begin{gathered}
\text { then } y=\frac{f\left(x_{c_{1}}\right) f\left(x_{c_{2}}\right)}{f\left(x_{c_{3}}\right) f\left(x_{c_{4}}\right)}+f\left(x_{c_{5}}\right) \text { with } C \\
f\left(x_{c_{r}}\right)=w_{0} x_{c_{r}}^{n}+w_{1} x_{c_{r}}^{n-1}+\ldots+w_{n-1} x_{c_{r}}+w_{n} \\
(i=1, \ldots, m),\left(x_{1}, \ldots, x_{c_{n}} \in \mathbf{X}\right), C \in[0,1]
\end{gathered}
$$

where $C$ is the certainty factor, $\mathbf{X}$ is the input set, $\mu_{k_{n}}$ are membership functions, and $w_{1}, \ldots, w_{n}$ are arithmetic expressions calculated during evolution. For expressing the fuzzy relations, Gaussian membership functions (MF) were selected. In this paper, we selected to use 3 MFs per attribute. Tuning of these MFs was also applied by evolutionary means. The selection of the variables in each rule is also guided by evolution. As fitness function, the mean square error (MSE) was selected. Table 1 summarizes the GP training parameters.

Table 1: Genetic programming training parameters.

\begin{tabular}{|c|c|}
\hline Parameter & Value \\
\hline GP System & Grammar-Guided GP \\
\hline Islands & 5 \\
\hline Island topology & Ring \\
\hline Isolation time & 50 generations \\
\hline Migrants number & 10 individuals \\
\hline Migrants type & Elite individuals \\
\hline Total population & 1,000 individuals \\
\hline Selection & Tournament \\
\hline Tournament size & 7 \\
\hline Elitism & Yes \\
\hline Crossover rate & 0.7 \\
\hline Mutation rate & 0.3 \\
\hline Max.individual size & 4,000 nodes \\
\hline Max.generations & 500 \\
\hline
\end{tabular}

\section{RESULTS AND DISCUSSION}

Our test results for the P1 and P2 systems in the Concrete Slump Test problem are shown in Table 2. Exact reconstruction of the test data set was not possible, as there is not related information in the literature (Yeh 08), however we selected to apply the same number of randomly chosen test records. Our results, in this example, are given in terms of Pearson correlation coefficient, to allow direct comparison with existing literature results. The highest reported accuracy in previous research, for this data set is $R^{2}=0.922$ (Yeh 08 ) where a neural network model was applied.

An example part of the derived rule base of the P1 system has the following form:

if Cement is Low then $y=1.4 x^{2}+1.5 x-9.3$,

(x: Water)

if Cement is Medium then $y=-2.3 x^{2}-0.2 x-8.1$,
Table 2: Results for the Concrete Slump Test.

\begin{tabular}{|c|c|c|}
\hline Parameter & P1 & P2 \\
\hline Size (nodes) & 2437 & 410 \\
\hline Size (rules) & 202 & 9 \\
\hline$R^{2}$ & 0.9127 & 0.80794 \\
\hline
\end{tabular}

(x: Coarse Aggr.)

if Water is Medium then $y=-1.4 x^{2}-1.7 x-3.3$,

(x: Fly Ash)

As it can be seen, in this problem, the P1 system compares well with the results reported in the literature, and in addition the output format allows for easier interpretation of the results. Although the P2 system achieved similar to P1 scores in our training and validation set, its performance for the test set was lower.

The test results for P1 and P2 systems for the Mackey-Glass data are summarised in Table 3.

Table 3: Results for the Mackey-Glass time series.

\begin{tabular}{|c|c|c|}
\hline Parameter & P1 & P2 \\
\hline Size (nodes) & 1867 & 457 \\
\hline Size (rules) & 98 & 8 \\
\hline MSE & 0.0033591 & 0.001336 \\
\hline
\end{tabular}

As expected, the higher order P2 model was able to provide a better approximation to this time-series. As an example output, one of the rules of the acquired solution by $\mathrm{P} 2$ system has the following form:

if $x_{4}$ is Low then $y=\frac{f_{1}\left(x_{3}\right) f_{2}\left(x_{3}\right)}{f_{3}\left(x_{4}\right) f_{4}\left(x_{3}\right)}+f_{5}\left(x_{2}\right)$ where:

$f_{1}\left(x_{3}\right)=1.14 x_{3}^{4}+0.15 x_{3}^{3}+0.44 x_{3}^{2}+0.24 x_{3}-0.23$,

$f_{2}\left(x_{3}\right)=-1.24 x_{3}^{4}+0.15 x_{3}^{3}+0.719 x_{3}^{2}+1.23 x_{3}+0.73$, $f_{3}\left(x_{4}\right)=0.42 x_{4}^{4}-0.36 x_{4}^{3}-1.22 x_{4}^{2}-0.74 x_{4}+1.11$, $f_{4}\left(x_{3}\right)=4.61 x_{3}^{4}-0.8 x_{3}^{3}+1.28 x_{3}^{2}-0.63 x_{3}-0.25$, $f_{5}\left(x_{2}\right)=-1.17 x_{2}^{4}+1.05 x_{2}^{3}+0.33 x_{2}^{2}-0.63 x_{2}+0.37$. ( $x_{2}$ : Slag, $x_{3}$ : Fly Ash, $x_{4}$ : Water).

Although direct comparison with previous literature results is not possible due to inability to reconstruct the exact training data set, past research is shown, for reference reasons, in Table 4 . As it can be seen in the table, our approach compares well to other models that do not employ local search methods.

\section{CONCLUSION AND FURTHER RESEARCH}

This paper presented a system for the generation of Takagi-Sugeno-Kang fuzzy rule based systems for regression and system identification, by means of genetic programming. The proposed approach carries several advantages over past related research. Firstly, 
Table 4: Results for the MacKey-Glass time series. Regression scores from (Jang 97), (Kim and Kim 97), (Wang 92) and (Lee and Kim 94).

\begin{tabular}{|c|c|}
\hline System & RMSE \\
\hline Linear regression model & 0.55 \\
\hline Auto regressive model & 0.19 \\
\hline Sixth order polynomial & 0.04 \\
\hline Back propagation NN & 0.02 \\
\hline GA and fuzzy system (5 MFs) & 0.049206 \\
\hline GA and fuzzy system (7 MFs) & 0.042275 \\
\hline GA and fuzzy system (9 MFs) & 0.037873 \\
\hline Wang Product T-norm & 0.907 \\
\hline Wang Min T-norm & 0.904 \\
\hline ANFIS & 0.007 \\
\hline P2-TSK-GP (3 MFs) - this paper & 0.036548 \\
\hline
\end{tabular}

the system is capable to automatically produce arbitrarily large and complex TSK fuzzy systems, according to the needs of a specific problem. Secondly, it provides flexibility in the selection of non-linear functions fired per rule. Finally, the output model is interpretable by humans in contrast to some other models like MLPs, since it is in the form of fuzzy rules. In this paper we have presented preliminary results of the experiments which while focusing on certain characteristics and capabilities of the GP algorithms produced encouraging results warranting further investigations.

Further research will be primarily focused on an advanced grammar design for efficient combinations of polynomials. Increasing the number of the membership functions is also expected to improve the accuracy of the system. Integration with ensemble systems will also be considered.

\section{ACKNOWLEDGEMENTS}

The research leading to these results has received funding from the European Commission within the Marie Curie Industry and Academia Partnerships and Pathways (IAPP) programme under grant agreement n. 251617.

\section{REFERENCES}

Alba E., Cotta C., Troya J.M., Evolutionary Design of Fuzzy Logic Controllers Using Strongly-Typed GP, In Proc. 1996 IEEE Int'l Symposium on Intelligent Control New York, NY., 127-132, 1996.

Augusto D.A., Barbosa H.J.C., Ebecken N.F.F., Coevolutionary Multi-population Genetic Program- ming for Data Classification GECCO'10, July 7-11, 2010, Portland, USA.

Blake C. L., Merz C. J., UCI repository of machine learning databases, http://www.ics.uci.edu/ mlearn/ML-Repository.html, 2001.

Chen Y., Yang B., Abraham A., Peng L., Automatic Design of Hierarchical Takagi-Sugeno Type Fuzzy Systems Using Evolutionary Algorithms IEEE Trans. on Fuzzy Systems , 15:3, 2007.

Delgado M.R., Zuben F.V., Gomide F., Coevolutionary genetic fuzzy systems: a hierarchical collaborative approach Fuzzy Sets and Systems, Elsevier, 141, 89-106, 2004.

Fernández F., Tommassini M.,Vanneschi L., An Empirical Study of Multipopulation Genetic Programming Genetic Programming and Evolvable Machines 4:1, Kluwer, 2003.

Hoffman F., Nelles O., Genetic Programming for Model Selection of TSK-Fuzzy Systems Information Sciences, Elsevier, 136, 7-28, 2001.

Jang J-S. R., Sun C.T., Mizutani E.,Neuro-Fuzzy and Soft Computing, Prentice Hall, NJ, 1997.

Jang J-S. R., Neuro-fuzzy modeling for nonlinear dynamic system identification in: Ruspini E. H. , Bonissone P. P. , Pedrycz W., eds., Handbook of fuzzy computation, Institute of Physics Publishing, Dirak House, Temple Back, Bristol, UK 1998.

Kim D., Kim C., Forecasting time series with genetic fuzzy predictor ensemble,IEEE Trans. Fuzzy Syst., 5:4, 523-535, 1997.

Koza J.R. Genetic programming - On the programming of computers by means of natural selection The MIT Press, Cambridge, Massachussets, USA, 1992.

Lee S.-H., Kim I., Time series analysis using fuzzy learning, inProc. Int'l Conf. Neural Inform. Process., Vol. 6, Seoul, Korea, 1577-1582, 1994.

Manderick B., Spiessens P., Fine-Grained Parallel Genetic Algorithms in Proc. of the 3rd Int'l Conf. on Genetic Algorithms, Morgan Kaufmann, 1989.

Montana D.J., Strongly Typed Genetic Programming, Evolutionary Computation, 3:2, 1995.

Wang L.X., Mendel J.M.,Generating fuzzy rules by learning from examples,IEEE Trans. Systems, Man and Cybernetics, 22:6, 1414-1427, 1992.

Yeh, I-C., Modeling slump of concrete with fly ash and superplasticizer, Computers and Concrete, 5:6, 559-572, 2008. 